DOI: $10.5455 / 2320-1770.1 j r \operatorname{cog} 20140354$

Case Report

\title{
Seroma: an interesting case report
}

\section{Hymavathi K. Reddy*, Ujwala J, Swetha M, Shilpa B. Ramya}

Department of Obstetrics \& Gynaecology, Narayana Medical College Hospital, Nellore, Andhra Pradesh, India

Received: 13 December 2013

Accepted: 06 January 2014

\section{*Correspondence:}

Dr. Hymavathi K. Reddy,

E-mail: drhymakrreddy@yahoo.co.in

(C) 2014 Reddy HK et al. This is an open-access article distributed under the terms of the Creative Commons Attribution Non-Commercial License, which permits unrestricted non-commercial use, distribution, and reproduction in any medium, provided the original work is properly cited.

\begin{abstract}
A seroma is defined as a sterile accumulation of serum in a circumscribed location in the tissue. Seromas can occur after surgeries, especially those that are extensive or involve significant tissue disruption viz. hernia repairs, plastic surgeries such as breast augmentation or reconstruction, abdominoplasties (tummy tucks) and removal of large intraabdominal space occupying lesions like huge fibroids/ovarian masses. The literature citing the exact incidence of seromas following removal of large intra-abdominal masses is scant. Seromas at times become severely symptomatic. The best way to prevent a seroma is not to give it any place to form. It's easier said than done. Here is an interesting case report of postoperative seroma following removal of a large broad ligament fibroid.
\end{abstract}

Keywords: Fibroid uterus, Abdominal hysterectomy, Seroma

\section{INTRODUCTION}

A seroma is an accumulation of fluid in a tissue or organ that can occur after surgery, or sometimes after an injury such as blunt trauma. The fluid, called serum, leaks out of nearby damaged blood and lymphatic vessels. Seromas can occur after surgeries, especially those that are extensive or involve significant tissue disruption. ${ }^{1,2}$ Seroma formation may be associated with an increased risk of infection and breakdown of the surgical site. ${ }^{3,4}$ Postoperative seromas may at times create enigma to the clinicians about a probable injury to a vital organ(?) during the course of surgery. We do hereby report an interesting case of seroma following the removal of a large broad ligament fibroid.

\section{CASE REPORT}

Mrs X, a short obese lady aged 40yrs has attended the Gynaec out-patient department of Narayana Medical College Hospital, a tertiary care centre with c/o intermittent dull lower abdominal pain on and off since two months, not associated with any fever / vomiting. H/o pain being relieved only after taking medication (anti spasmodics) at times. There were no urinary or bowel disturbances. She is a known hypertensive on treatment. Her menstrual cycles were regular with moderate flow. She was para 2 , live 2, both were full term, caesarean section deliveries and was tubectomised. Past history: similar painful episodes infrequently since two years for which she has consulted an outside Gynaecologist and was advised surgery-hysterectomy.

\section{On examination}

She was obese with BMI-31. Mild anaemiat. Heart \& lungs-NAD.

Per abdominal examination: Fatty abdominal wall with a midline subumbilical vertical puckered scar (previous $\mathrm{C} / \mathrm{S}$ scar). A firm irregular non-tender mass of $20 \times 20 \mathrm{cms}$ felt on the left side extending from hypogastrium to left lumbar region. Only the right border of the lump could be vaguely made out. Mobility of the lump could not be appreciated. No other positive findings were present. Per speculum examination: Cervix visualization was difficult and was drawn up. Per vaginal examination: Cervix felt high up and behind the pubic symphysis. Lower part of abdominal mass was felt through anterior, posterior and left lateral fornices with irregular nodularity and firm 
consistency. Uterus could not be made out separately. Provisional diagnosis: Large uterine fibroid/ovarian mass.

\section{Ultrasonogram}

Bulky uterus $(13.2 \times 4.5 \times 8.6 \mathrm{cms})$ with anterior intramural fibroid $(4.5 \times 5 \mathrm{cms})$. Large mass lesion $(17 \times 15 \times 10 \mathrm{cms})$ is noted arising from left uterine wall extending from pelvis extending to left lumbar region. The lesion is heterogenous, hypointense with vascularity on CDFI. This mass lesion is seen displacing the bowel loops and indenting left external Iliac vessels. Rt \& Lt. Ovaries normal in size and echo texture. No E/O hydronephrosis.

\section{Impression}

Large subserosal/Broad ligament fibroid. Suggested CECT abdomen. Patient could not get CECT done, due to financial problems.

The couple was counseled for surgery - Exploratory laparotomy. Following routine surgical workup with special investigations like Thyroid profile and CA125 (WNL) case posted for exploratory laparotomy. In view of suspected large broad ligament fibroid, pre-operative Urological consultation was done for retrograde Ureteric stenting.

\section{Operative findings}

Abdomen opened by midline vertical incision through previous C.S. scar. Dense adhesions b/n parietal peritoneum, omentum and surrounding bowel loops \& Uterus + . Uterus enlarged to 12-14 weeks size. A big Fibroid $(20 \times 17 \mathrm{Cms})$ was found originating from left side of the body of uterus extending laterally \& upwards almost to the hypochondriac region. Both the leaves of the left broad ligament were stretched over the fibroid. It was deeply impacted in the left paracolic gutter. Right ovary enlarged (5.5x4 Cms), cystic \& unhealthy. Urinary bladder was drawn up \& densely adherent to lower part of uterine body. Following the adhesiolysis, Fibroid was disimpacted from it's base and proceeded with hysterectomy \& bilateral salpingooophorectomy. Clamping \& ligation of pedicles on the left side was difficult due to the fibroid size. Bladder separation was very difficult $\&$ there was troublesome active ooze during separation. The procedure was done up to the clamping of uterine vessels taking all the precautions to avoid ureteric inclusion into the clamps. Due to technical difficulty as well as active bleeding during bladder separation with impending threat of bladder injury, decided for subtotal hysterectomy \& proceeded with. Following verification of haemostasis, abdomen closed leaving intraperitoneal \& subcutaneous drains in situ. Tension sutures were applied due to obese abdominal wall. Postoperative period was uneventful. Drains removed on $5^{\text {th }}$ postoperative day (Intra peritoneal drain $<20 \mathrm{ml} \&$ subcutaneous drain $<15 \mathrm{ml} / 24 \mathrm{Hrs}$ ). Patient got discharged against advice on $7^{\text {th }}$ postoperative day.
Patient got readmitted after 3 days $\left(10^{\text {th }}\right.$ postoperative day) with c/o pain abdomen, vomiting \& loose stools of one day duration. On examination there were no significant findings. Serum electrolytes were WNL. She was kept on NPO \& parenteral antibiotics (Ofloxacin \& metronidazole) along with I.V. fluids. She felt better with relief of her complaints within 24 hours. On 13th postoperative day suture removal was done pending the tension suture removal for the next day. Wound healed well. The same day evening she $\mathrm{C} / \mathrm{O}$ discharge of watery liquid from the drain site on left side which was found to be partially gaped \& leaking of watery liquid was observed (This intra peritoneal drain was removed on $5^{\text {th }}$ postoperative day itself). Subsequently the amount of watery discharge became profuse. Ultrasound abdomen revealed moderate amounts of fluid collection in peritoneal cavity. Foley's catheter introduced \& left in situ to monitor urine output. Urological consultation was done suspecting a ureteric leak. Advised to do creatinine (Cr) estimation in both serum \& watery discharge from the drain site. Cr. levels were found to be almost same. Urine output was normal $(1700 \mathrm{ml})$. Fluid leakage is still profuse wetting the dressing pads $\&$ bed sheets $\left(15^{\text {th }}\right.$ Post op.) But there was no urinary smell in leaking fluid. Case reviewed by Urologist, advised to give Tab. Pyridium 2 stat orally and observe colour change of both Urine \& leaking fluid. Few hours after pyridium administration, urine in the bag was found to slightly orange coloured \& progressively became dark orange by evening. Leaking fluid remained clear and the amount of leaking also found to be gradually reducing and stopped almost completely by $18^{\text {th }}$ postoperative day. Thus the diagnosis of "Seroma" is confirmed. Foley's removed and there were no more complaints. Patient was discharged from the hospital on $20^{\text {th }}$ P.O. day.

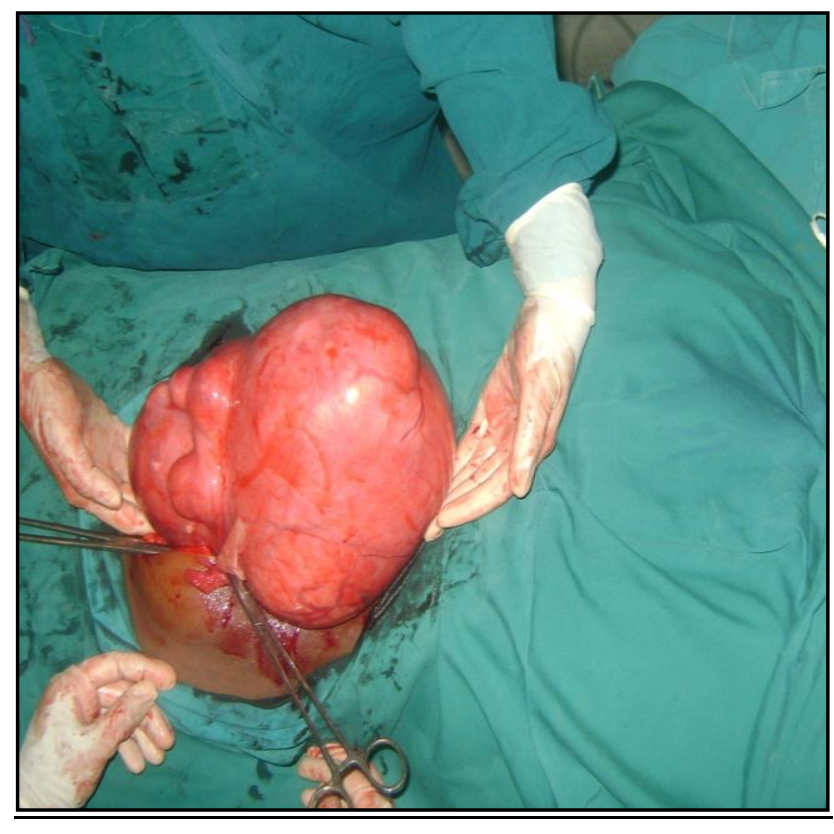

Figure 1: Large broad ligament fibroid (Intraoperative view). 


\section{DISCUSSION}

A seroma is defined as a sterile accumulation of serum beneath the tissue in the area of surgical incision. Seromas can occur after different types of surgeries, especially those that are extensive or involve significant tissue disruption. ${ }^{1,2}$ These include hernia repairs, significant plastic surgeries such as breast augmentation or reconstruction, abdominoplasties (tummy tucks), ${ }^{5,6}$ and removal of large intra-abdominal masses like huge fibroids/ovarian masses as in our case. Seroma formation may be associated with an increased risk of infection and breakdown of the surgical site.

The difference between a seroma and an abscess is that an abscess involves the presence of white blood cells, bacteria, and the breakdown products of both. In other words, an abscess is defined as an infection. A seroma, on the other hand, is just fluid, serum that has accumulated in a dead space in the tissue. It is the result of tissue insult and the product of tissue inflammation and the body's defense mechanisms. The body is simply reacting to the presence of a dead space within the tissue that was previously attached to something. When a large mass is removed as in our case - a big fibroid, damage to the very small vessels that previously ran from the underlying tissue (i.e., muscle, connective tissue) to the overlying tissue can occur. Although these vessels do not cause significant blood loss, they do allow escape of serum into the area. This may be also due to the resulting tissue damage that occurs regardless of how carefully the dissection is done. This tissue damage results in cellular death. The body's reaction is an inflammatory one. ${ }^{7}$ Because of the inflammation, cell death, and increased vascular permeability, fluid can accumulate in the newly created space. This process will generally resolve over time if there is some form of natural drainage or sufficient circulation to the area and if the person is in good health. A seroma is mainly a nuisance but not dangerous. Sometimes it may be necessary to intervene to try and do something about the problem.

The first and best way is to prevent a seroma formation by not giving it any place to form. One precaution to be taken is gentle handling of the tissues to the possible extent. This would mean either using fingers or a blunt instrument to tear the underlying tissue. Tearing the tissue causes the small vessels to vasoconstrict, thereby stopping any bleeding. The use of clamps, suture or cautery may also be effective in sealing the small bleeders. In some cases pressure application over the surgical site for 3-4 days following surgery may also help in preventing fluid accumulation. This can be done by closure of the tissues intra operatively taking care not to leave any dead space or post operatively by using tight bandages over that site. Drains that are typically placed at the time of surgery also help reducing seroma formation. ${ }^{7}$ The drainage is done not only to allow the surfaces to stay in contact and stick together but also to prevent potential infection or creation of a chronic pocket (bursa). Surgical drain tubes with bulb suction devices are used after some surgeries to help reduce the risk of seroma formation. These allow for monitoring the volume of fluid leakage, and once drainage becomes minimal, the drains are removed. In our case, as there was a large dead space following fibroid removal, precautions were taken by putting intraperitoneal \& subcutaneous drains to prevent any fluid collection post operatively in the respective places. Apart from putting the drains, tight supportive abdominal bandage was applied in immediate post-operative period as an adjunctive preventive measure against fluid accumulation.

Seromas can form shortly after surgery if drains are not used, and they may also occur after removal of a drain. In our case both intra peritoneal and subcutaneous drains were removed on $5^{\text {th }}$ P.O. day after the drainage fluid was found to be less than $15 \mathrm{ml}$ for two consecutive days. In the index case, it is evident that seroma formation occurred after a considerable time following drain removal.

Small seromas can be persistent for a very long time (even years). When seroma becomes a clinical concern it should first be treated conservatively. Increasing the circulation to the healing area will often help to reduce the swelling. The fluid will be re-absorbed into the blood stream faster and the increased blood flow will bring oxygen and nutrients to the newly forming tissue. Heat is an excellent way to increase circulation to an area. Hot packing is a simple inexpensive and a very effective way to conservatively manage a seroma.

In symptomatic seromas (ones seen or felt) the first thing to be done is to do percutaneous aspiration and sometimes everything will be settled, but unfortunately seromas may reform as the dead space and the cavity will not be obliterated by just aspiration. Obliteration of dead space with various flap fixation techniques, use of sclerosants, fibrin glue and sealants, octreotide, and pressure garments have been attempted with conflicting results and none have been consistent. ${ }^{8}$ Once the aspirated volume is around $10 \mathrm{cc}$ or less aspirations can be stopped. They usually "burn out" so to speak after repeated aspirations. In the index case, the resultant seroma became symptomatic by spontaneously leaking out through the previous drain site. In that way, aspirations were not needed $\&$ the continuous leakage for nearly 2 days resulted in its burning out.

If a seroma remains for too long over the span of several weeks it will form a bursa or sac around it. ${ }^{9}$ If this happens, the seroma will not be absorbed and may become infected. The best approach for this might be to open up the area surgically and drain the seroma with a drain, excising the bursal sac entirely. ${ }^{10}$ This rarely is required.

To summarize: In our case, the significant features are obese lady with history of two previous caesarean section deliveries, and a long standing large broad ligament fibroid. Having anticipated intraoperative problems, pre- 
operative retrograde ureteric stenting was done. During surgery ample care was taken to avoid ureteric inclusion in the clamps \& measures for perfect haemostasis were observed throughout, with careful tissue handling. Intraperitoneal \& subcutaneous drains along with tight abdominal pressure bandage were implemented to prevent post-operative fluid collection. Smooth Immediate post-operative period later followed by leakage of clear watery liquid through drain site made us suspicious of left ureteric injury (clamping/cautery). As seromas are rarely encountered by the Gynaecologists our thoughts didn't go in that direction and the worry was mainly about the ureteric injury. Ultimately it was the seroma that caused the whole menace.

\section{CONCLUSION}

Though seromas are rarely reported following gynaecological surgeries, it is suggested that one should always keep in mind of a possible occurrence of a seroma following removal of large space occupying pelvic tumours, so that preventive measures can be taken both during and after the surgeries.

Funding: No funding sources Conflict of interest: None declared

Ethical approval: Not required

\section{REFERENCES}

1. Better Medicine. Seroma - causes, symptoms, treatment - better medicine, 2013. Available at: www.localhealth.com/article/seroma.

2. V. D. Pinchuk, O. V. Tymofii, Gusztáv Gulyás. Commentary on "Seroma as a Late Complication after Breast Augmentation" Aesthetic Plastic Surgery. 2011 June;35(3):315-8.

3. Wikipedia, the encyclopedia. Seroma, 2011. Available at: en.wikipedia.org/wiki/Seroma.

4. Natalie L. Semchyshyn, Dirk M. Elston. Dermatologic Surgical Complications. Available at: http://medisuite.ir/medscape/a1128404business.html.

5. Venkataram, Jayashree. "Tumescent liposuction: A review." J Cutan Aesthet Surg. 2008 JulDec;1(2):49-57.

6. USA Food and Drug Administration - Consumer Health Information. "The Skinny on Liposuction," $2010 . \quad$ Available at: http://www.fda.gov/consumer/updates/liposuction08 2007.html. Accessed 20 January 2010.

7. Data Sciences International. Scientific Services PRWeb, 2012. Available at: www.prweb.com/releases/2012/9/prweb9910371.ht m. Accessed 18 September 2012.

8. Vivek Srivastava, Somprakas Basu, Vijay Kumar Shukla. Seroma formation after breast cancer surgery: what we have learned in the last two decades breast cancer. 2012 December;15(4):373-80.

9. Right Diagnosis. Right diagnosis from health grades, 2013. Available at: www.rightdiagnosis.com/seroma/treatments. Accessed 4 December 2013.

10. Letty liposuction directory. All about liposuction, 2008. Available at: www.letty-liposuctiondirectory.com.

DOI: $10.5455 / 2320-1770 . i j \operatorname{cog} 20140354$

Cite this article as: Reddy HK, Ujwala J, Swetha M, Ramya SB. Seroma: an interesting case report. Int J Reprod Contracept Obstet Gynecol 2014;3:254-7. 